\title{
Model of Finite-Momentum Excitons Driven by Surface Plasmons in Photoexcited Carbon Nanotubes Covered by Gold Metal Films
}

\author{
Seiji Uryu, ${ }^{1, *}$ Hiroshi Ajiki, ${ }^{2}$ and Hajime Ishihara $^{3}$ \\ ${ }^{1}$ Department of Materials Science and Engineering, Iwate University, 4-3-5 Ueda, Morioka, Iwate 020-8551, Japan \\ ${ }^{2}$ Photon Pioneers Center, Osaka University, 2-8 Yamadaoka, Suita, Osaka 565-0871, Japan \\ ${ }^{3}$ Department of Physics and Electronics, Osaka Prefecture University, 1-1 Gakuencho, Nakaku, Sakai, Osaka 599-8531, Japan
}

(Received 21 December 2012; published 18 June 2013)

\begin{abstract}
Optical spectra of finite-momentum excitons in carbon nanotubes with gold nanostructures are theoretically studied. A Green function method is developed for self-consistently solving Maxwell equations including the quantum-mechanical nonlocal response of the nanotubes and the local response of the nanostructures. Excitons with finite momenta in the axis direction in the nanotubes are effectively excited by localized electric fields due to surface plasmons in the gold nanostructures and counteract the surface plasmons through depolarization fields, showing the crucial self-consistency of these effects.
\end{abstract}

Optically excited electrons and holes in semiconductors and insulators can be bound to excitons. Since the wave vector of light is usually much smaller than the reciprocal lattice vectors of solids, exciton momenta transferred from light are negligible. However, finite-momentum excitons have been studied because of their important roles in the optical properties [1-3]. Light irradiation onto metal nanostructures causes surface plasmon resonance accompanying localized electric fields with a large intensity and large wave vectors, which have intensively been used to enhance the optical response of materials near the nanostructures such as surface-enhanced Raman scattering [4]. On the other hand, because of large wave vectors of the fields, finite-momentum excitons in low-dimensional bulks are expected to be excited near the nanostructures.

Excitation of finite-momentum excitons provides a nonlocal response since they contribute to the conductivity with finite wave vectors, i.e., nonlocal conductivity. Position-dependent current induces charge and counteracting electric fields called depolarization fields occur even in bulk systems, affecting the surroundings. From the theoretical point of view, calculation of nonlocal response of materials and the resulting response of the surroundings is challenging [5]. In this study, we theoretically propose the spectroscopy of finite-momentum excitons in semiconducting carbon nanotubes by the surface plasmons of gold nanostructures, which could be a typical example of a spectroscopic means of revealing excitation processes that cannot be addressed by conventional methods.

Semiconducting carbon nanotubes are quasi-onedimensional bulk systems exhibiting the most significant exciton effects [6-15]. Normally, light cannot excite excitons with finite longitudinal momenta in the tube axis direction. For light polarization parallel and perpendicular to the axis, excitons with zero and finite angular momenta about the axis, respectively, are excited. For the latter, depolarization fields occur, which generally reduce exciton effects and increase exciton energies and electric fields at the increased energies [8,10,16-18]. The excitons for parallel and perpendicular polarization are denoted as $E_{i i}$ and $E_{12}$ excitons, respectively, with $i=1,2, \ldots$ indicating associated sub-bands. So far, in carbon nanotubes with metal nanostructures, surface-enhanced Raman scattering [19], photoluminescence [20,21], and antenna effects $[22,23]$ have been studied. Metal structures with gaps of several nanometers [24-26] are suitable for the excitation of finite-momentum excitons in nanotubes.

In calculations for carbon nanotubes with metal nanostructures, the nonlocal response must be considered for the nanotubes, while for the metal nanostructures with the size more than a few tens nanometers, the local response adequately describes surface plasmons [27]. Thus, we shall consider the wave equation for the electric field $\mathbf{E}_{\omega}(\mathbf{r})$ at position $\mathbf{r}$ and frequency $\omega$,

$$
\begin{aligned}
{[\nabla} & \left.\times \nabla \times-k_{0}^{2}-4 \pi i k_{0} \sigma_{\omega}(\mathbf{r})\right] \mathbf{E}_{\omega}(\mathbf{r}) \\
& =4 \pi i k_{0} \int d \mathbf{r}^{\prime} \sigma_{\omega}\left(\mathbf{r}, \mathbf{r}^{\prime}\right) \mathbf{E}_{\omega}\left(\mathbf{r}^{\prime}\right),
\end{aligned}
$$

where $k_{0}=\omega / c$ with the speed of light $c$ and $\sigma_{\omega}(\mathbf{r})$ and $\sigma_{\omega}\left(\mathbf{r}, \mathbf{r}^{\prime}\right)$ are the local and nonlocal conductivity tensors, respectively. We develop a simple and general method to numerically solve Eq. (1). Discretizing space, Eq. (1) becomes

$$
\left(D-k_{0}^{2} I-4 \pi i k_{0} \sigma_{\omega}^{L}\right) \mathbf{E}_{\omega}=4 \pi i k_{0} \sigma_{\omega}^{N} V \mathbf{E}_{\omega},
$$

where $\mathbf{E}_{\omega}$ is an electric field vector whose dimensions are thrice the number of discrete positions, $D$ a matrix describing $\nabla \times \nabla \times, \sigma_{\omega}^{L}$ and $\sigma_{\omega}^{N}$ matrices for $\sigma_{\omega}(\mathbf{r})$ and $\sigma_{\omega}\left(\mathbf{r}, \mathbf{r}^{\prime}\right)$, respectively, $V$ a diagonal matrix of volume elements, and $I$ a unit matrix.

The field in Eq. (2) is a solution of the equation,

$$
\mathbf{E}_{\omega}=\mathbf{E}_{\omega}^{(1)}+4 \pi i k_{0} G_{\omega} V \sigma_{\omega}^{N} V \mathbf{E}_{\omega}
$$


where $\mathbf{E}_{\omega}^{(1)}$ is a field without the nonlocal response materials and the Green function $G_{\omega}$ is defined as

$$
\left(D-k_{0}^{2} I-4 \pi i k_{0} \sigma_{\omega}^{L}\right) G_{\omega} V=I .
$$

Since Eq. (3) is closed for positions for nonlocal response, it can be solved. From Eq. (4),

$G_{\omega}=G_{\omega}^{(0)}+4 \pi i k_{0} G_{\omega}^{(0)} V \sigma_{\omega}^{L}\left[I-4 \pi i k_{0} G_{\omega}^{(0)} V \sigma_{\omega}^{L}\right]^{-1} G_{\omega}^{(0)}$,

where $G_{\omega}^{(0)}$ is the Green function in vacuum, defined as $\left(D-k_{0}^{2} I\right) G_{\omega}^{(0)} V=I$. The field $\mathbf{E}_{\omega}^{(1)}$ is given by

$$
\mathbf{E}_{\omega}^{(1)}=\mathbf{E}_{\omega}^{(0)}+4 \pi i k_{0} G_{\omega}^{(0)} V \sigma_{\omega}^{L} \mathbf{E}_{\omega}^{(1)},
$$

where $\mathbf{E}_{\omega}^{(0)}$ is an incident field [28]. In Eqs. (3) and (6), the second terms on the right-hand side are depolarization fields, where $G_{\omega}$ and $G_{\omega}^{(0)}$, respectively, describe the propagation of the fields. Since an analytical dyadic Green function can be used for $G_{\omega}^{(0)}$, only materials are discretized. Another method for the Green functions similar to $G_{\omega}$ has been reported [29].

The electronic states of the carbon nanotubes are described in the lowest-order effective-mass scheme, where interaction effects on the band structure are considered in the screened Hartree-Fock approximation and excitons are described by the superposition of electronhole pair states [6]. Note that the equation of motion for electron-hole pairs has a screened attractive interaction but not an exchange interaction describing depolarization effects because the above formalism includes them [30]. The nonlocal conductivity for nanotubes is given by the Kubo formula $[6,16]$ and a model of position-independent local conductivity [31] is used for the gold nanostructures.

The depolarization effects in a nanotube can be qualitatively understood by the continuity equation, $-\omega \rho+\mathbf{k} \cdot \mathbf{j}=0$, where $\rho$ and $\mathbf{j}$ are the charge and current densities, respectively, and $\mathbf{k}$ a wave vector. For $E_{12}$ excitons, because $\mathbf{j}$ is nearly in the circumference direction, depolarization fields occur for zero longitudinal momentum owing to the finite $\rho$ for angular momenta but the effects of the longitudinal momenta are small. For $E_{i i}$ excitons, however, finite longitudinal momenta cause longitudinal depolarization effects since $\mathbf{j}$ is almost along the axis, which are characteristic of bulk systems. Thus, longitudinal momenta enhance energy dispersions for $E_{i i}$ excitons but not for $E_{12}$ excitons.

We choose a specific system in Figs. 1(a) and 1(b) as a typical example that causes strong fields localized at a narrow gap and is realized [25]. However, the qualitative features of the following results are general. In the figures, two square gold blocks, each with side length $W$ and thickness $W / 3$, are located with gap width $G$. The lower side of the blocks is at $z=0$. The nanotube is in the $x y$ plane where its axis is at $z=z_{0}(<0)$ and directed along $\mathbf{e}_{\|}$ or $\mathbf{e}_{\perp}$ in Fig. 1(a). Incident light polarized linearly along $\mathbf{e}_{\|}$propagates in the $z$ direction. A background dielectric constant $\kappa$ of 2.6 is used [32].

Figure 1 shows the typical surface-plasmon-induced localized fields near a gap at $z=z_{0}$ as the components along (c) $\mathbf{e}_{\|}$and (d) the $z$ axis for $W=60 \mathrm{~nm}$ without the nanotube at the light energy $E=1.44 \mathrm{eV}$. For $z<0$, the field vanishes at $|z| \gg G$, and the $z$ component is negligible at $z \approx 0$, because of the field directed in the $x y$ plane, and takes a maximum at $|z| \sim G$. For nanotubes along $\mathbf{e}_{\|}$and $\mathbf{e}_{\perp}, E_{i i}$ and $E_{12}$ excitons, respectively, can be excited by almost the same field that is along $\mathbf{e}_{\|}$and localized in the tube axis direction. This fact is convenient for analysis. Nanotubes along $\mathbf{e}_{\|}$can also excite $E_{12}$ excitons. In the following, $E_{22}$ and $E_{12}$ excitons are considered because their energies, which depend on the diameter, are close to the surface plasmon resonances.

Figure 2 shows the typical absorption spectra of a nanotube with length $A=113 \mathrm{~nm}$ and blocks with $W=60 \mathrm{~nm}$ for nanotubes along (a) $\mathbf{e}_{\|}$and (b) $\mathbf{e}_{\perp}$ where (a) $E_{22}$ and (b) $E_{12}$ excitons are at the surface plasmon resonance. The results are separately plotted for absorption in the tube region and that in the block region. Dotted and dashed lines show the results for setting $G_{\omega}$ in Eq. (3) to zero and to $G_{\omega}^{(0)}$ for $\sigma_{\omega}^{L} \rightarrow 0$ in Eq. (5), respectively, and solid lines those for full calculations. Then, depolarization effects are neglected for the dotted lines and those of the nanotubes do not include effects of the blocks for the dashed lines.

For a nanotube in Fig. 2(a), exciton peaks appear at $E=1.43 \mathrm{eV}$. As compared to the peaks for the dotted line, those for the dashed and solid lines are broader with
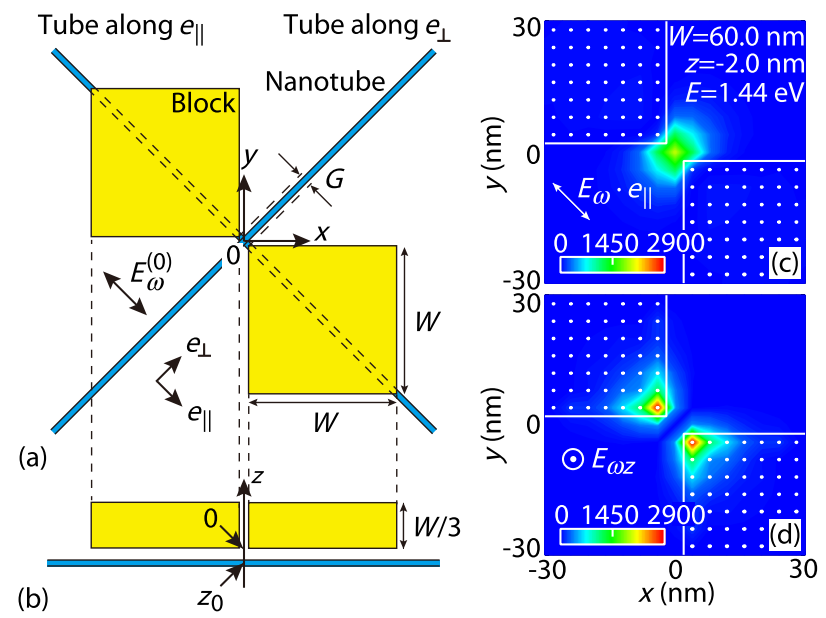

FIG. 1 (color online). Schematic illustrations of (a) top and (b) side views of a system. The nanotube is discretized by squares with sides $L / 8$, where $L$ is the circumference length and the blocks by cubes with sides $W / 15 . G=(\sqrt{2} / 15) W \cdot z_{0}=$ $-W / 30$. Squared absolute values of the field components along (c) $\mathbf{e}_{\|}$and (d) the $z$ axis at $z=z_{0}$ in units of the intensity of incident light. Dots enclosed by the solid lines are projections of the discretized points for blocks. 


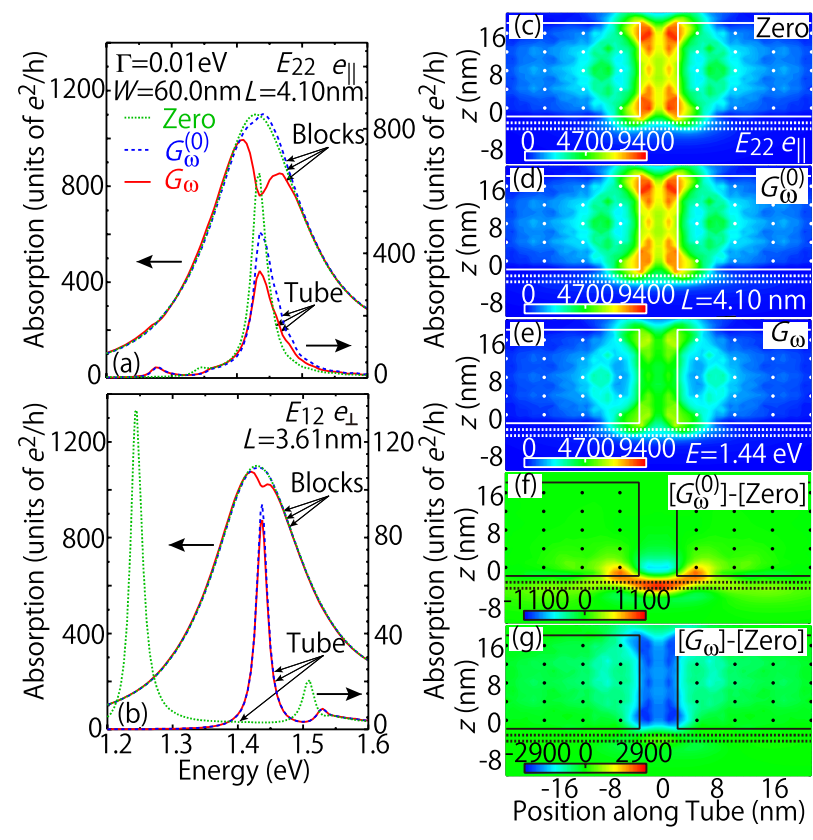

FIG. 2 (color online). Absorption spectra of blocks and nanotubes with $L$ being (a) 4.10 and (b) $3.61 \mathrm{~nm}$ for (a) $E_{22}$ and (b) $E_{12}$ excitons, showing the light-energy dependence of an absorption coefficient $C$ defined by $P=C\left[E_{\omega}^{(0)}\right]^{2} / 2 W^{2}$ with the absorption power $P$ and amplitude of incident light $E_{\omega}^{(0)}$. See text for definitions of lines. Scales for the blocks and nanotubes are at the left and right vertical axes, respectively. Phenomenological energy broadening for the nanotubes $\Gamma=0.01 \mathrm{eV}$ is used. (c)-(g) Field intensities for (a) in units of incident light intensity. See text for definitions of panels. Dots enclosed by solid lines are discretized points for blocks and dotted lines nanotubes.

enhanced tails on the high-energy side because of the longitudinal depolarization effect as mentioned before, showing the excitation of finite longitudinal-momentum excitons. For the blocks, broad peaks appear because of surface plasmon resonance. Only the solid line shows a dip at the peak for the nanotube. This is due to the fields reduced at the gap by the self-consistency of the depolarization fields resulting from the finite-momentum excitons and the response of the blocks to the fields, which $G_{\omega}$ accurately describes. Thus, the dip is another evidence for the excitation of finite-momentum excitons.

This dip is understood to be analogous to interaction between two small materials, 1 and 2, where separation between them is much smaller than the wavelength of light and 1 has discrete resonant levels. For simplicity, they are considered to have only a diagonal local conductivity component vertical to the line connecting them, $\sigma_{i}$, where $i=\{1,2\}$. From Eq. (6), for an initial field in the direction of $\sigma_{i}$, equations for the same components of fields averaged over each material, $E_{1}$ and $E_{2}$, are $\alpha_{11} E_{1}+\alpha_{12} E_{2}=$ $E_{0}$ and $\alpha_{21} E_{1}+\alpha_{22} E_{2}=E_{0}$ where $E_{0}$ is the initial field which is the same for 1 and $2, \alpha_{i j}=\delta_{i j}-4 \pi i k_{0} G_{i j} v_{j} \sigma_{j}$ with the Green function between $i$ and $j, G_{i j}$, which is real and satisfies $G_{11}<G_{21}<0$, and the volume of $j, v_{j}$. Near the $l$ th level of $1, \varepsilon_{l}, \sigma_{1} \approx-i a_{l}\left(\varepsilon_{l}-\hbar \omega\right)^{-1}$ with a positive real number $a_{l}$. At $\hbar \omega$ slightly above $\varepsilon_{l}, \alpha_{11}<\alpha_{21}<0$, while at $\hbar \omega$ slightly below $\varepsilon_{l+1}\left(>\varepsilon_{l}\right), \alpha_{11}>\alpha_{21}>0$. Thus, there exists $\hbar \omega_{l}$ where $\alpha_{11}=\alpha_{21}$ between $\varepsilon_{l}$ and $\varepsilon_{l+1}$. At $\hbar \omega_{l}, E_{2}=0$, i.e., absorption vanishes for 2 .

Recent results that the local response of small molecules near metal nanostructures causes dips in the plasmon peaks $[33,34]$ are considered to have the same origin. However, our results are essentially different from the others in that interaction between a nanotube and blocks arises from the nonlocal response of the nanotube. For the dips for $E_{i i}$ excitons, the field parallel to the tube axis needs to be strongly localized in the axis direction to cause a strong longitudinal depolarization field.

For a clearer demonstration of the above, the field intensity near the gap in a plane through the tube and $z$ axes at $E=1.44 \mathrm{eV}$ is shown in Figs. 2(c)-2(g). The result for the dotted lines in Fig. 2(a) is Fig. 2(c). Those for the dashed and solid lines in Fig. 2(a) are Figs. 2(d) and 2(e), respectively, and differences of Figs. 2(d) and 2(e) from Fig. 2(c) are Figs. 2(f) and 2(g), respectively. Finitemomentum excitons enhance the field around the nanotube near the gap as shown in Fig. 2(f), but response of the blocks to the field finally reduces the field at the gap as shown in Fig. 2(g).

Figure 2(b) is qualitatively the same as Fig. 2(a) except for the following: peaks for the nanotube shift from 1.25 to $1.43 \mathrm{eV}$ because of the depolarization effect for the angular momenta [16] and tails of the peaks for the dashed and solid lines are negligible because of weak longitudinal depolarization effects as mentioned before. The dip in the solid line for the blocks mainly arises from the angular momenta which is smaller than that in Fig. 2(a) because of the smaller oscillator strength of $E_{12}$ excitons.

Figure 3(a) shows the absorption spectra of the nanotubes (solid lines) and blocks (dashed lines) for various block sizes for nanotubes along $\mathbf{e}_{\|}$, where $E_{22}$ excitons are at the surface plasmon resonances. The dotted lines indicate the absorption of the whole system, i.e., sum of the results for the solid and dashed lines. All the solid lines show exciton peaks, which are reflected as dips in the plasmon peaks of the dashed lines. With increasing $W$, the dip becomes smaller. The dips for the dashed and dotted lines appear up to $W \sim 100$ and $\sim 30 \mathrm{~nm}$, respectively. Figure 3(b) shows the differential cross section for backward scattering for the cases of Fig. 3(a). Only the absorption of the blocks is well pronounced since the volume of the nanotubes is negligibly smaller than that of the blocks.

In the following, we propose how to measure the energy dispersions of the excitons. The inset of Fig. 4(c) shows a system where a nanotube is under a gold block array with period $T$ and separation $G$ in the array direction. Irradiation by light linearly polarized in the array direction causes 


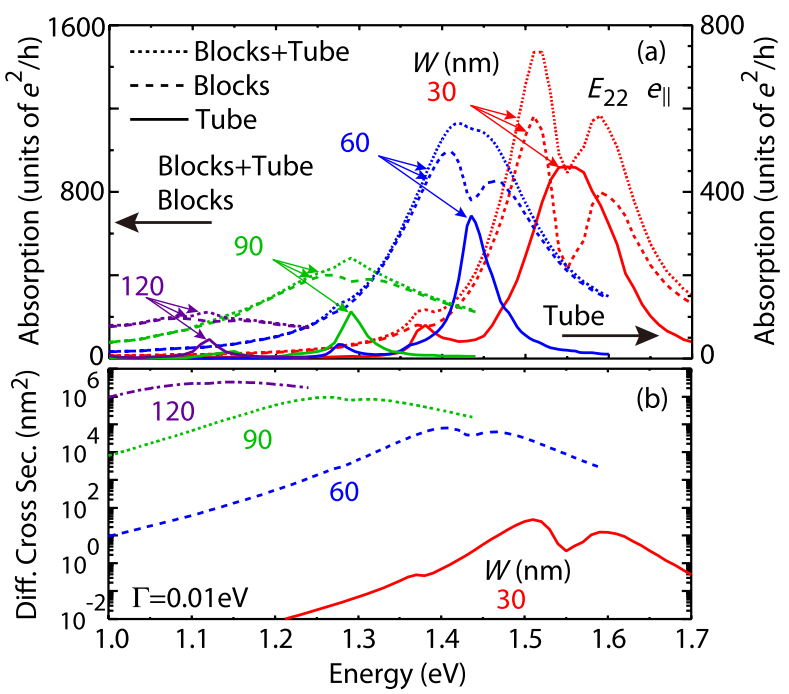

FIG. 3 (color online). (a) Absorption spectra and (b) differential cross section for backward scattering for $E_{22}$ excitons. $(W, L)=(30,3.68),(60,3.78),(90,4.10)$, and $(120$, 4.59) nm. $A=113 \mathrm{~nm} . \Gamma=0.01 \mathrm{eV}$. In (a), see text for definitions of lines. Scales for the dashed and dotted lines are at the left vertical axis and scales for the solid lines are at the right one. In (b), $W=30$ (solid line), 60 (dashed line), 90 (dotted line), and $120 \mathrm{~nm}$ (dot-dashed line).

periodic localized light near the gaps with wave vectors $2 \pi n / T$ in the array direction with integers $n$. Thus, excitons with the same longitudinal wave vectors are selectively excited depending on their energies. Not only $E_{22}$ but also $E_{12}$ excitons can be excited since the $z$ component of the fields is similar to that in Fig. 1(d). This geometry is preferred as it is more easily realized than periodic arrays of that in Fig. 2.

Figure 4 shows the absorption spectra of a nanotube (solid lines), blocks (dashed lines), and the whole system (dotted lines) per period for (a) $E_{22}$ and (b) $E_{12}$ excitons. In Fig. 4(a), the solid line shows a clear oscillation with the maxima denoted by vertical arrows, showing the excitation of excitons with wave vectors $2 \pi n / T$ for $n=0,1,2$, and 3 . The dashed line shows a plasmon peak with dips reflecting the exciton peaks. The results in Fig. 4(b) are similar except for a smaller oscillation period resulting from a narrower dispersion. Dispersions of $E_{22}$ and $E_{12}$ excitons derived from Figs. 4(a) and 4(b) are plotted in Fig. 4(c) by filled circles and crosses, respectively. Solid and dashed lines denote dispersions of $E_{22}$ and $E_{12}$ excitons, respectively, without the blocks. The results for the symbols well agree with those for the lines. The small deviations are possibly due to the screening of depolarization fields by the blocks and the coupling of the excitons with the surface plasmons.

The spectra for nanotubes and blocks in this study can be separately obtained. The differential cross section and electric field intensity at a gap [35] as shown in Figs. 3(b) and 2(e), respectively, could be the spectra of
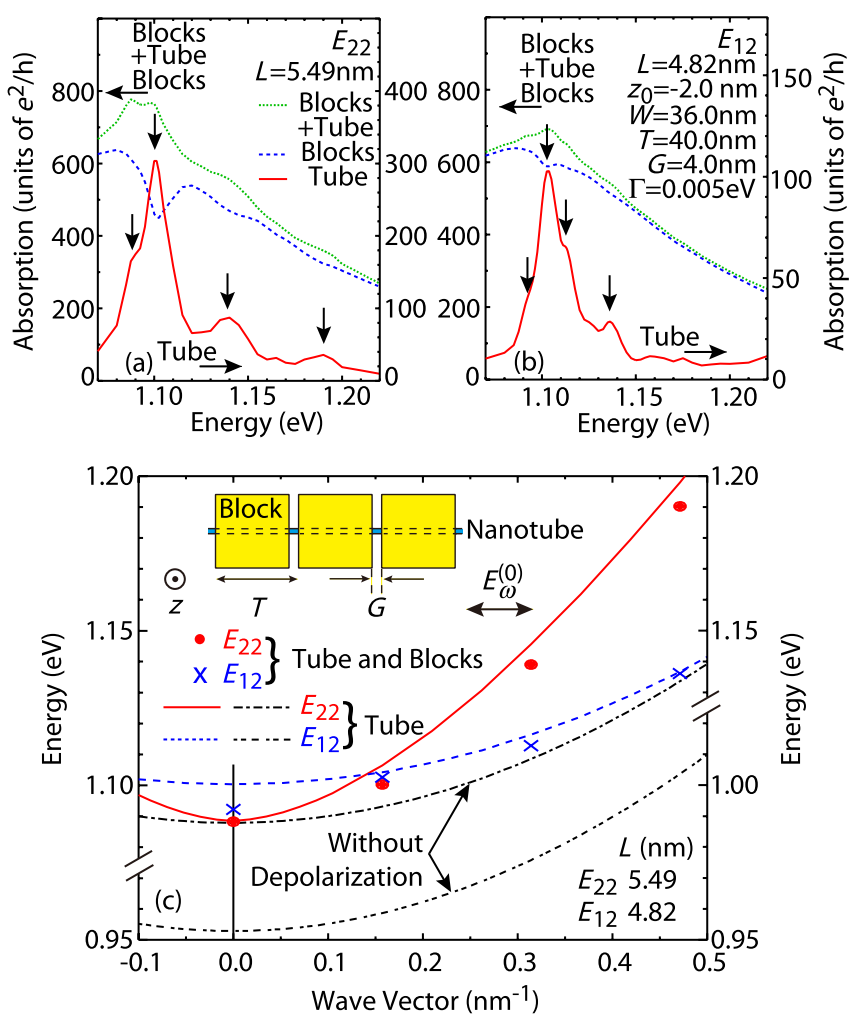

FIG. 4 (color online). Absorption spectra for (a) $E_{22}$ and (b) $E_{12}$ excitons with $L$ being (a) 5.49 and (b) $4.82 \mathrm{~nm} . z_{0}=$ $-2 \mathrm{~nm} .(W, T, G)=(36,40,4) \mathrm{nm} . \Gamma=0.005 \mathrm{eV}$. See text for definitions of lines. The blocks are discretized by $W / 9$. (c) Dispersions of $E_{22}$ (circles) and $E_{12}$ (crosses) excitons. Lines are those without blocks where solid and dashed lines denote $E_{22}$ and $E_{12}$ excitons, respectively, and dot-dashed and double-dot-dashed lines $E_{22}$ and $E_{12}$ excitons, respectively, without depolarization effects. Inset is a schematic illustration of a system.

the blocks. For the nanotubes, it is beneficial to obtain photoluminescence-excitation spectra. Excited states in the blocks decay nonradiatively while those in the nanotubes first decay into the lowest $E_{11}$ excitons nonradiatively and then decay by emitting light whose energy is independent of the excitation energy. Thus, the emission intensity as a function of the excitation energy would constitute the absorption spectra of the nanotubes only. Suppression of emission due to energy transfer of $E_{11}$ excitons to the blocks is not severe because this emission is observable [20,21].

Our method is useful in that it enables accurate selfconsistent calculations including both local and quantummechanical nonlocal responses. We often require such calculations since description of materials by local response functions can greatly simplify problems as compared to the use of nonlocal response functions. The method is applicable to the spectroscopies of crystal surfaces and molecules, biosensors, etc., with metal and dielectric nanostructures. 
In conclusion, the optical response of carbon nanotubes with gold nanostructures has been theoretically studied. Finite longitudinal momentum excitons in the nanotubes are effectively excited by surface plasmons in the nanostructures, which appear in the spectra of both the nanotubes and nanostructures because of depolarization effects. Spectroscopy using localized electric fields will further help in revealing excited states that are inaccessible by conventional methods.

This work was supported in part by the Grants-in-Aid for Scientific Research No. 19049014 on Priority Area "Strong Photons-Molecules Coupling Fields" from the Ministry of Education, Culture, Sports, Science and Technology, Japan and the Japan Society for Promotion of Science. A part of the numerical calculations was performed at Iwate University Super Computing and Information Sciences Center.

*uryu@iwate-u.ac.jp

[1] R. G. Ulbrich and C. Weisbuch, Phys. Rev. Lett. 38, 865 (1977).

[2] F. DeMartini, M. Colocci, S.E. Kohn, and Y.R. Shen, Phys. Rev. Lett. 38, 1223 (1977).

[3] G. Göger, M. Betz, A. Leitenstorfer, M. Bichler, W. Wegscheider, and G. Abstreiter, Phys. Rev. Lett. 84, 5812 (2000).

[4] M. Moskovits, Rev. Mod. Phys. 57, 783 (1985) and references therein.

[5] K. Cho, Optical Response of Nanostructures: Microscopic Nonlocal Theory (Springer, Berlin, 2003).

[6] T. Ando, J. Phys. Soc. Jpn. 66, 1066 (1997).

[7] C.D. Spataru, S. Ismail-Beigi, L.X. Benedict, and S. G. Louie, Phys. Rev. Lett. 92, 077402 (2004).

[8] E. Chang, G. Bussi, A. Ruini, and E. Molinari, Phys. Rev. Lett. 92, 196401 (2004).

[9] V. Perebeinos, J. Tersoff, and Ph. Avouris, Phys. Rev. Lett. 92, 257402 (2004).

[10] H. Zhao and S. Mazumdar, Phys. Rev. Lett. 93, 157402 (2004).

[11] C.L. Kane and E. J. Mele, Phys. Rev. Lett. 93, 197402 (2004).

[12] M. Ichida, S. Mizuno, Y. Tani, Y. Saito, and A. Nakamura, J. Phys. Soc. Jpn. 68, 3131 (1999).
[13] S. M. Bachilo, M.S. Strano, C. Kittrell, R. H. Hauge, R.E. Smalley, and R. B. Weisman, Science 298, 2361 (2002).

[14] F. Wang, G. Dukovic, L. E. Brus, and T. F. Heinz, Science 308, 838 (2005).

[15] J. Maultzsch, R. Pomraenke, S. Reich, E. Chang, D. Prezzi, A. Ruini, E. Molinari, M. S. Strano, C. Thomsen, and C. Lienau, Phys. Rev. B 72, 241402 (2005).

[16] S. Uryu and T. Ando, Phys. Rev. B 74, 155411 (2006).

[17] Y. Miyauchi, M. Oba, and S. Maruyama, Phys. Rev. B 74, 205440 (2006).

[18] J. Lefebvre and P. Finnie, Phys. Rev. Lett. 98, 167406 (2007).

[19] X. Hou and Y. Fang, Spectrochim. Acta, Part A 69, 1140 (2008).

[20] T. Sakashita, Y. Miyauchi, K. Matsuda, and Y. Kanemitsu, Appl. Phys. Lett. 97, 063110 (2010).

[21] G. Hong, S. M. Tabakman, K. Welsher, H. Wang, X. Wang, and H. Dai, J. Am. Chem. Soc. 132, 15920 (2010).

[22] J. Waxenegger, A. Trügler, and U. Hohenester, Phys. Rev. B 83, 245446 (2011).

[23] N. Rauhut, M. Engel, M. Steiner, R. Krupke, Ph. Avouris, and A. Hartschuh, ACS Nano 6, 6416 (2012).

[24] Y. Sawai, B. Takimoto, H. Nabika, K. Ajito, and K. Murakoshi, J. Am. Chem. Soc. 129, 1658 (2007).

[25] K. Ueno, S. Juodkazis, T. Shibuya, Y. Yokota, V. Mizeikis, K. Sasaki, and H. Misawa, J. Am. Chem. Soc. 130, 6928 (2008).

[26] H. Duan, A. I. Fernández-Domínguez, M. Bosman, S. A. Maier, and J. K. W. Yang, Nano Lett. 12, 1683 (2012).

[27] J. M. McMahon, S. K. Gray, and G. C. Schatz, Phys. Rev. Lett. 103, 097403 (2009).

[28] T. Iida and H. Ishihara, Physica (Amsterdam) 26E, 163 (2005).

[29] F. J. García de Abajo and A. Howie, Phys. Rev. B 65, 115418 (2002).

[30] H. Ajiki, T. Iida, T. Ishikawa, S. Uryu, and H. Ishihara, Phys. Rev. B 80, 115437 (2009).

[31] F. Hao and P. Nordlander, Chem. Phys. Lett. 446, 115 (2007).

[32] T. Ando, J. Phys. Soc. Jpn. 73, 3351 (2004).

[33] G. L. Liu, Y.-T. Long, Y. Choi, T. Kang, and L. P. Lee, Nat. Methods 4, 1015 (2007).

[34] H. Ishihara, A. Nobuhiro, M. Nakatani, and Y. Mizumoto, J. Photochem. Photobiol., A 221, 148 (2011).

[35] Y. Tanaka, H. Ishiguro, H. Fujiwara, Y. Yokota, K. Ueno, H. Misawa, and K. Sasaki, Opt. Express 19, 7726 (2011). 